\title{
Evaluation of Urine as a Diagnostic Specimen for Visceral Leishmaniasis in Sudan
}

\author{
Emma van Rij ${ }^{1}$, Bakri Y M Nour² and Henk D F H Schallig ${ }^{1 *}$ \\ ${ }^{1}$ Koninklijk Insituut voor de Tropen (KIT) / Royal Tropical Institute, KIT Biomedical Research, Parasitology Unit, Netherlands \\ ${ }^{2}$ Blue Nile National Institute for Communicable Diseases, Department of Parasitology, University of Gezira, Sudan
}

\begin{abstract}
Diagnostic methods for visceral leishmaniasis $(V L)$ require invasive specimen sampling. Urine is a potential noninvasive alternative and in the present study the diagnostic performance of direct agglutination test (DAT), based on a freeze dried antigen, and rK39 strip test (InBios, Bio-Rad) on specimens collected in Sudan was assessed. RK39 test had a sensitivity of $72.1 \%$ and a specificity of $76.9 \%$ on urine and DAT sensitivity was $62.8 \%$ and its specificity $69.2 \%$, using initial diagnosis (VL diagnosis was confirmed on clinical and serological basis) as reference in both cases. Tests agreements were fair. Both rK39 as well DAT have potential in diagnosing VL using urine, but results are currently not as good as on the Indian sub-continent.
\end{abstract}

Keywords: Visceral leishmaniasis; Diagnosis; Urine; Sudan

\section{Introduction}

The diagnosis of visceral leishmaniasis (VL) can either be direct via the demonstration by culture of microscopy of Leishmania parasites in lymph node, bone marrow or spleen aspirates or indirectly through serology or molecular biology [1]. Direct demonstration is invasive and requires technical expertise and a certain high level of medical precautions, in particular in the case of splenic aspiration (often considered to be the reference standard), as this has the risk of serious bleeding. Molecular biology is not yet field applicable and therefore serological tests, like the direct agglutination test (DAT) or rK39 immunochromatographic rapid tests are often employed as diagnostic methods [1]. However, serology still requires collection of blood samples which has an inherent risk of accidents, is a taboo in some countries and resented by some groups such as small children. Therefore, research into alternative diagnostic methods is not only looking for better applicable techniques, but also for patient samples that can be obtained in a non-invasive manner, such as urine.

There are several reports, in particular from the Indian subcontinent that describes a good diagnostic performance of rK39 strip tests using urine samples [2-4]. In contrast, limited information on testing of urine samples with serology is available from East Africa (Sudan and Ethiopia), another VL hot spot, although it is well known that both tests have an overall good sensitivity and specificity in that particular region when performed on serum samples; DAT: $94.23 \%$ and $89.97 \%$, respectively and rK39: $94.48 \%$ and $88.75 \%$, respectively [5].

Therefore, we initiated a preliminary evaluation of rk39 strip tests and DAT on urine samples collected in Sudan, which is reported here.

\section{Materials and Methods}

Urine samples were collected from individuals in the catchment area of Umkoraa Rural Hospital in West of Gedarif State and Al Azaza Damus Kala-azar Clinic in Sennar State, which is an endemic area for malaria with seasonal transmission, and one of the most important VL areas in the world. Patients were diagnosed at recruitment in Sudan for malaria based on clinical symptoms and light microscopy of a thin blood smear, and were included in this study when the blood smear was positive for asexual parasites, identified as P. falciparum. VL diagnosis was based on clinical symptoms (including irregular fever, weight loss, splenomegaly and hepatomegaly) and serological testing; unfortunately parasitological testing was not performed. VL cases were included when a positive DAT on serum was obtained, with a titre $>1: 1600$, or when DAT titre was 1:1600 and rK39 Kalazar Detect Rapid Test (InBios, International Inc., USA) or IT-Leish (Bio-Rad, France) strip test on serum was positive. The presence of a malaria infection in these cases was excluded through microscopy on a blood smear. This resulted in a total of $26 \mathrm{VL}$ patients, 18 malaria patients and 17 patients with malaria-VL co-infections to be included in the study. The samples were kept on ice during transportation, and were stored without preservative at $-20^{\circ} \mathrm{C}$ at KIT, Amsterdam, Netherlands.

In addition, 3 samples from apparently endemic healthy controls (EHCs) from Sudan and 5 urine samples from non-endemic healthy controls (NEHC), in the Netherlands, were included in the study. In the laboratory, urine samples were tested with the rK 39 Kalazar Detect Rapid Test, using an adapted protocol [2]. In brief, $150 \mu$ of urine was applied directly to the test strip, without chasing buffer, and results were examined after $10 \mathrm{~min}$. A test was considered positive when both the control line and the test line were visible, and negative when only the control line was visible. If the control line was not visible, a test was considered invalid.

The protocol of the FD-DAT, manufactured by KIT (Amsterdam, the Netherlands), was adapted for the use of urine. A $100 \mu$ l whole urine sample was added to the first row of a 96-well plate, and was two-fold serially diluted with $50 \mu \mathrm{l}$ of saline+ $0.1 \mathrm{M} \beta$-mercaptoethanol. $50 \mu \mathrm{l}$ of reconstituted freeze-dried Leishmania antigen was added to each well, and results were examined the next day. Each plate contained a positive or negative control. Results were interpreted as negative when only a blue dot was visible and positive when agglutination was observed, with a cut-off value of 1:8 (based on prior testing with urine samples

*Corresponding author: Henk D F H Schallig, Koninklijk Insituut voor de Tropen (KIT) / Royal Tropical Institute, KIT Biomedical Research, Parasitology Unit, Netherlands, Tel: +31-20-5665447; Fax: +31-20-6971841; E-mail: h.schallig@kit.n

Received May 01, 20123; Accepted May 29, 2013; Published May 31, 2012

Citation: Van Rij E, Nour BYM, Schallig HDFH (2013) Evaluation of Urine as a Diagnostic Specimen for Visceral Leishmaniasis in Sudan. J Trop Dis 1: 106 doi:10.4172/329-891X.1000106

Copyright: ( 2013 Van Rij E, et al. This is an open-access article distributed unde the terms of the Creative Commons Attribution License, which permits unrestricted use, distribution, and reproduction in any medium, provided the original author and source are credited. 
from NEHC). The agreement between both diagnostic test and initial clinical diagnosis or between DAT and rK39 on urine in general was determined by calculating Kappa $(\mathrm{k})$ values with $95 \%$ confidence intervals using Epi-info version 6 [6].

\section{Results and Discussion}

The results of testing urine samples with rK39 strip test or DAT for VL are presented in table 1 . The rk39 test had a sensitivity of $72.1 \%$ (95\% CI: $57.3-83.3$ ) and a specificity of $76.9 \%$ (95\% CI: $58.9-88.9$ ) using the initial diagnosis (clinical symptoms combined with positive serology) as reference. The sensitivity of DAT on urine was $62.8 \%(95 \%$ CI: 47.9-75.6) and specificity $69.2 \%(50.0-83.5)$.These sensitivities and specificities are lower than those observed on the Indian sub-continent [2-4], but are better than those of another urine based diagnostic test, KAtex, in East Africa [7]. The difference between Indian and East African level of specificity and sensitivity could be explained by the heterogeneity of $L$. donavani strains in East Africa versus their homogeneity in India [7].

It is noted that the agreement between the general performance of DAT and rK39 on urine samples is good (Table 2). The present study has some limitations. First, the total number of cases studies is low, but the work is intended as a pilot study to assess whether there is some potential for urine testing for VL diagnosis. A follow-up study will include more cases and all case definition must be improved, in particular with respect to the VL cases. The urine samples have been stored without a preservative and transported prior to analysis and this might have affected tests' performance [3]. Interestingly, rK39 and DAT performed almost equally on urine samples in the present study.
This is in contrast to studies using serum samples from East Africa in which the rK39 test is reported to have a lower sensitivity and specificity [7]. In particular it is noted that several urine samples of malaria cases were also found positive, which cannot be explained at this stage, although it might be possible that these patients have an underlying asymptomatic leishmaniasis infection, as was demonstrated in India [8], which could contribute to this positive reaction with urine.

In conclusion, the present study has demonstrated some potential of diagnostic testing of urine with DAT and rk39 test for VL, but efforts to further improve test performance should be undertaken. Furthermore, it would be of interest to assess if, and how many (percentage) urine samples stay positive after a complete cure (as a possible test of cure).

\section{Author's contributions}

EvR conducted diagnostic tests in the Netherlands and drafted the manuscript; BYMN collected field samples, performed initial diagnostic analysis in Sudan and commented on the manuscript; HDFHS designed the study, supervised diagnostic testing in the Netherlands and finalized the manuscript.

\section{Acknowledgements}

We are grateful to study staff and patients at Umkoraa Rural Hospital and A Azaza Damus Kala-azar Clinic for their participation in the study.

\section{Ethical Approval}

The study was approved by the National Health Research Ethics Committee National Ministry of Health Sudan (study protocol 140-11-10). A written informed consent was obtained from every Sudanese subject included in the study. Dutch urine samples were obtained with oral informed consent from volunteers.

\begin{tabular}{|c|c|c|c|c|}
\hline \multicolumn{3}{|l|}{ A. } & DAT & rK39 \\
\hline \multicolumn{3}{|l|}{ Healthy Endemic Controls } & $1 / 3$ & $0 / 3$ \\
\hline \multicolumn{3}{|l|}{ Healthy Non-endemic Controls } & $0 / 5$ & $1 / 5$ \\
\hline \multicolumn{3}{|l|}{ Confirmed Malaria Cases } & $7 / 18$ & $5 / 18$ \\
\hline \multicolumn{3}{|c|}{ Confirmed Visceral Leishmaniasis Cases } & $18 / 26$ & $19 / 26$ \\
\hline \multicolumn{3}{|c|}{ Confirmed VL - Malaria co-infections } & 9/17 & $12 / 17$ \\
\hline \multicolumn{5}{|c|}{ The numbers in the section A above represents: the number of samples found positive by the DAT or rK39 test/total number of urine samples tested } \\
\hline B. & $\%$ Agreement & $\kappa$ value & $95 \% \mathrm{Cl}$ & Agreement \\
\hline $\begin{array}{l}\text { rK39 on urine compared to } \\
\text { diagnosis }\end{array}$ & $73.9 \%$ & 0.469 & $0.262-0.676$ & "moderate" \\
\hline $\begin{array}{l}\text { DAT on urine compared to } \\
\text { diagnosis }\end{array}$ & $65.2 \%$ & 0.302 & $0.083-0.521$ & "fair" \\
\hline $\begin{array}{l}\text { rK39 on serum compared to } \\
\text { DAT on serum }\end{array}$ & $81.2 \%$ & 0.631 & $0.460-0.803$ & "good" \\
\hline $\begin{array}{l}\text { Rk39 on urine compared to } \\
\text { rK39 on serum }\end{array}$ & $68.1 \%$ & 0.367 & $0.151-0.582$ & "fair" \\
\hline $\begin{array}{l}\text { DAT on urine compared to } \\
\text { DAT on serum }\end{array}$ & $63.7 \%$ & 0.237 & $0.051-0.469$ & "fair" \\
\hline
\end{tabular}

In section B the agreement between the different test/diagnosis is presented. The agreement between the tests was determined by calculating Kappa values with $95 \%$ confidence intervals using Epi-info version 6 . Kappa values express the agreement beyond chance ${ }^{6}$.

Table 1: Results of urine testing for visceral leishmaniasis with rK39 strip test and DAT.

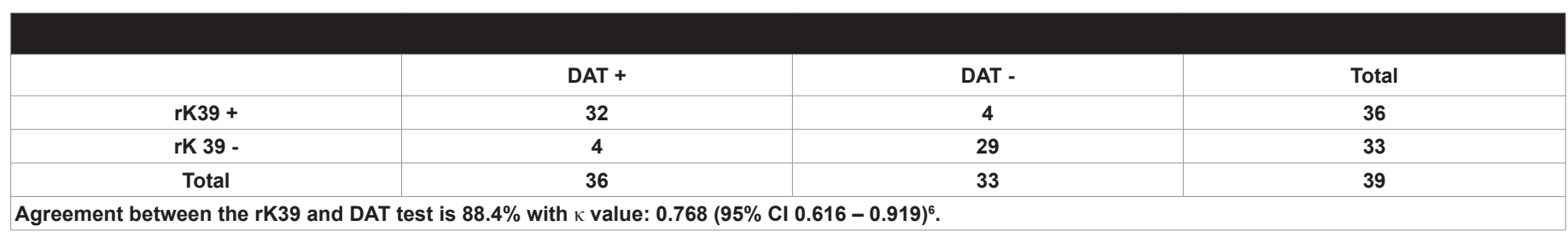

Table 2: The agreement between rK39 and DAT testing of urine samples. 
Citation: Van Rij E, Nour BYM, Schallig HDFH (2013) Evaluation of Urine as a Diagnostic Specimen for Visceral Leishmaniasis in Sudan. J Trop Dis 1: 106. doi:10.4172/329-891X.1000106

Page 3 of 3

\section{References}

1. World Health Organization on behalf of the Special Programme for Research and Training in Tropical Diseases (2011) Diagnostic evaluation series No. 4. Visceral leishmaniasis rapid diagnostic test performance. Geneva: World Health Organization.

2. Chakravarty J, Kumar S, Kumar R, Gautam S, Rai M, et al. (2011) Evaluation of rk39 immunochromatographic te.st with urine for diagnosis of visceral leishmaniasis. Trans R Soc Trop Med Hyg 105: 537-539.

3. Khan MG, Alam MS, Podder MP, Itoh M, Jamil KM, et al. (2010) Evaluation of rK-39 strip test using urine for diagnosis of visceral leishmaniasis in an endemic area in Bangladesh. Parasit Vectors 3: 114.

4. Singh D, Pandey K, Das VN, Das S, Verma N, et al. (2013) Evaluation of rK39 strip test using urine for diagnosis of visceral leishmaniasis in an endemic region of India. Am J Trop Med Hyg 88: 222-226.
5. Maia Z, Lírio M, Mistro S, Mendes CM, Mehta SR, et al. (2012) Comparative study of rK39 Leishmania antigen for serodiagnosis of visceral leishmaniasis: systematic review with meta-analysis. PLoS Negl Trop Dis 6: e1484.

6. Altman DG (1991)Practical Statistics for Medical Research. Chapman \& Hall, London, UK.

7. Boelaert M, El-Safi S, Hailu A, Mukhtar M, Rijal S, et al. (2008) Diagnostic tests for kala-azar: a multi-centre study of the freeze-dried DAT, rK39 strip test and KAtex in East Africa and the Indian subcontinent. Trans R Soc Trop Med Hyg 102: $32-40$.

8. Hasker E, Kansal S, Malaviya P, Gidwani K, Picado A, et al. (2013) Latent infection with Leishmania donovani in highly endemic villages in Bihar, India PLoS Negl Trop Dis 7: 2053. 